\title{
The Pseudo Orbit Tracing Properties on the Space of Probability Measures
}

\author{
Motomasa KOMURO \\ Tokyo Metropolitan University \\ (Communicated by K. Ogiue)
}

\section{Introduction}

Let $X$ be a state space of some system and $M(X)$ the space of probability measures on $X$. The elements of $M(X)$ are viewed as statistical states. The elements of $X$ are imbedded in $M(X)$ as the pure states. Let $T$ be a transformation of $X$ and $\widetilde{T}$ the corresponding transformotion of $M(X)$. As against $(X, T)$ is a dynamical system in classical mechanics, $(M(X), \widetilde{T})$ can be viewed as one in classical statistical mechanics (cf. [1]).

If $X$ is compact metric and $T$ is continuous, then $M(X)$, provided with the weak topology, is again compact metric and $\widetilde{T}$ is continuous. W. Bauer and K. Sigmund studied in [1] the problem of which of the properties of $(X, T)$ (like distality, topologically mixing, expansiveness, etc...) carry over to $(M(X), \widetilde{T})$. However they did not treat the pseudo orbit tracing property defined by R. Bowen [2]. The aim of this paper is to study the property of $(M(X), \widetilde{T})$ induced by $(X, T)$ which has the pseudo orbit tracing property.

\section{§1. Definitions and results.}

Let $X$ be a compact metric space with metric $d$ and $M(X)$ the space of Borel probability measures on $X$. The Prohorov metric $\widetilde{d}$ on $M(X)$ is defined by $\widetilde{d}(\mu, \nu)=\inf \left\{\varepsilon: \mu(A) \leqq \nu\left(A^{*}\right)+\varepsilon\right.$ and $\nu(A) \leqq \mu\left(A^{*}\right)+\varepsilon$ for all Borel sets $A \subset X\}$ for $\mu, \nu \in M(X)$. Here $A^{\varepsilon}=\cup_{x \in A}\{y \in X: d(x, y) \leqq \varepsilon\}$. As V. Strassen showed in [7], one has $\widetilde{d}(\mu, \nu)=\inf \left\{\varepsilon: \mu(A) \leqq \nu\left(A^{*}\right)+\varepsilon\right.$ for all Borel sets $A \subset X$ \}. The induced topology is just the weak topology for measures. It turns $M(X)$ into a compact space (cf. [5, P. 45]). For $x \in X$, let $\pi(x) \in$ $M(X)$ be a point measure defined by $\pi(x)(A)=1$ if $x \in A,=0$ otherwise.

Received August 12, 1983.

Revised January 19, 1984. 
$\pi$ is a homeomorphism from $X$ onto a closed subset of $M(X)$. It is obvious that $M(X)$ is convex and the point measures are just the extremal points of $M(X)$. Let $M_{n}(X)=\left\{\mu=(1 / n)\left(\pi\left(x_{1}\right)+\cdots+\pi\left(x_{n}\right)\right), x_{i} \in X\right.$ not necessarily distinct for $n=1,2, \cdots$. Then $M_{n}(X)$ is a closed subset of $M(X)$ and $\cup_{n \geq 1} M_{n}(X)$, the measures with finite support, is dense in $M(X)$.

Let $T$ be a homeomorphism of $X$ (i.e. from $X$ onto itself). $T$ induces a map $\widetilde{T}: M(X) \rightarrow M(X)$ defined by $(\widetilde{T} \mu)(A)=\mu\left(T^{-1} A\right) \quad(\mu \in M(X), A \subset X$; Borel). It is easy to see that $\widetilde{T}$ is a homeomorphism of $M(X)$, sending $\pi(x)$ into $\pi(T x)$. Clearly $M_{n}(X)(n \geqq 1)$ is $\widetilde{T}$-invariant. We denote the restriction of $\widetilde{T}$ to $M_{n}(X)$ by same symbol $\widetilde{T}$. It is well known that the set of $T$-invariant measures $\{\mu \in M(X): \widetilde{T} \mu=\mu\}$, which is just the set of fixed points of $\widetilde{T}$, is a nonempty convex closed set ([3, P. 17]). Let $X_{n}=X \times \cdots \times X$ ( $n$-times) and a metric $d_{n}$ on $X_{n}$ define by $d_{n}(x, y)=$ $\max _{1 \leq i \leq n} d\left(x_{i}, y_{i}\right)$ for $x=\left(x_{1}, \cdots, x_{n}\right), y=\left(y_{1}, \cdots, y_{n}\right)$ in $X_{n}$. $T$ induces a homeomorphism $T_{n}$ of $X_{n}$ defined by $T_{n}(x)=\left(T x_{1}, \cdots, T x_{n}\right) \quad(x=$ $\left.\left(x_{1}, \cdots, x_{n}\right) \in X_{n}\right)$.

A sequence $\left\{x_{i}\right\}_{i=a}^{b}(-\infty \leqq a \leqq b \leqq \infty)$ in $X$ is $\delta$-pseudo orbit of $T$ if $d\left(T x_{i}, x_{i+1}\right)<\delta$ for $a \leqq i \leqq b-1$. The $\delta$-pseudo orbit $\left\{x_{i}\right\}_{i=a}^{b}$ is said to be $\varepsilon$ traced if there is $x \in X$ with $d\left(T^{i} x, x_{i}\right) \leqq \varepsilon(a \leqq i \leqq b) . \quad(X, T)$ has the pseudo orbit tracing property (abbrev. P.O.T.P.) if for every $\varepsilon>0$ there is $\delta>0$ such that every $\delta$-pseudo orbit of $T$ is $\varepsilon$-traced. $(X, T)$ is tracing if for a sequence $\left\{x_{i}\right\}_{i=0}^{\infty}$ with $\lim _{i \rightarrow \infty} d\left(T x_{i}, x_{i+1}\right)=0$ there is $x \in X$ with $\lim _{i \rightarrow \infty} d\left(T^{i} x, x_{i}\right)=0$. In general the tracing does not imply the P.O.T.P.. $(X, T)$ is said to be $T$-connected if for every $x, y \in X$ and every $\alpha>0$ there are $\alpha$-pseudo orbits $\left\{x_{i}\right\}_{i=0}^{a}$ and $\left\{y_{i}\right\}_{i=0}^{b}$ so that $x_{0}=x=y_{b}$ and $y_{0}=y=x_{a}$. $(X, T)$ is topologically mixing if for every nonempty open sets $U$ and $V$ of $X$ there is $N>0$ such that $U \cap T^{-n} V \neq \varnothing$ for every $n \geqq N . \quad(X, T)$ satisfies specification if for every $\varepsilon>0$ there is $M=M(\varepsilon)>0$ such that for every $k \geqq 1$ and $k$ points $x_{1}, \cdots, x_{k} \in X$, for every set of integers $a_{1} \leqq$ $b_{1}<\cdots<a_{k} \leqq b_{k}$ with $a_{i+1}-b_{i} \geqq M(1 \leqq i \leqq k-1)$ and for every $p \geqq b_{k}-a_{1}+M$ there is $x \in X$ with $d\left(T^{n} x, T^{n} x_{i}\right) \leqq \varepsilon\left(a_{i} \leqq n \leqq b_{i}, 1 \leqq i \leqq k-1\right)$ and $T^{p} x=x$. $(X, T)$ satisfies weak specification if $(X, T)$ satisfies the definition of specification except the periodic condition; $T^{p} x=x$. Our results are following.

THEOREM 1. Let $T$ be a homeomorphism of a compact metric space $X$ and $\widetilde{T}$ an induced homeomorphism of $M(X)$. Then the following holds.

(1) If $(X, T)$ has the P.O.T.P., then $\left(M_{n}(X), \widetilde{T}\right)$ has also the P.O.T.P. for every $n \geqq 1$. 
(2) If $(X, T)$ is tracing, then $\left(M_{n}(X), \widetilde{T}\right)$ is also tracing for every $n \geqq 1$.

THEOREM 2. Let $\tilde{T}$ be as in Theorem 1. The following holds.

(1) If $(M(X), \widetilde{T})$ is tracing, then $(M(X), \widetilde{T})$ has the P.O.T.P..

(2) If $(M(X), \widetilde{T})$ has the P.O.T.P., then $(M(X), \widetilde{T})$ satisfies specification.

REMARK. By Theorem 2(2) it follows that if $(M(X), \widetilde{T})$ has the P.O.T.P. (resp. tracing), then $(X, T)$ is topologically mixing (cf. Propositions 21.3 and 6.9 in [3]). There is $(X, T)$ which has the P.O.T.P. (resp. tracing) but is not topologically mixing (for example $X=\{0,1\}$ and $T=i d$.), so $(M(X), \widetilde{T})$ need not have the P.O.T.P. (resp. tracing) even if $(X, T)$ has the P.O.T.P. (resp. tracing).

\section{§2. Proof of Theorem 1 .}

For $n \geqq 1$, let us define a map $\varphi_{n}: X_{n} \rightarrow M_{n}(X)$ by $\varphi_{n}(x)=(1 / n) \sum_{i=1}^{n} \pi\left(x_{i}\right)$ $\left(\boldsymbol{x}=\left(x_{1}, \cdots, x_{n}\right) \in X_{n}\right)$. Clearly $\varphi_{n}$ is a continuous surjection at most $n$ ! to one. Moreover $\varphi_{n}$ satisfies following;

LEMMA 1. For every $\boldsymbol{x}, \boldsymbol{y} \in X_{n}, \tilde{d}\left(\varphi_{n}(\boldsymbol{x}), \varphi_{n}(\boldsymbol{y})\right) \leqq d_{n}(\boldsymbol{x}, \boldsymbol{y})$.

ProOF. Let $\boldsymbol{x}=\left(x_{1}, \cdots, x_{n}\right), \boldsymbol{y}=\left(y_{1}, \cdots, y_{n}\right) \in X_{n}$ be given. Put $d_{n}(\boldsymbol{x}, \boldsymbol{y})=c$, then $d\left(x_{i}, y_{i}\right) \leqq c(1 \leqq i \leqq n)$. For every Borel set $A \subset X$ if $y_{i} \in A$ then $x_{i} \in A^{\circ}$. Hence we have $\varphi_{n}(\boldsymbol{x})\left(A^{\circ}\right) \geqq \varphi_{n}(\boldsymbol{y})(A)$, and so by the definition of $\widetilde{d}, \widetilde{d}\left(\varphi_{n}(\boldsymbol{x}), \varphi_{n}(\boldsymbol{y})\right) \leqq c=d_{n}(\boldsymbol{x}, \boldsymbol{y})$.

LEMMA 2 (marriage lemma [4]). Let $B=\left\{b_{1}, \cdots, b_{n}\right\}$ and $G=\left\{g_{1}, \cdots, g_{n}\right\}$ be finite sets of cardinal $n$ and $P(G)$ the family of subsets of $G$. Let $\Psi$ be a map from $B$ into $P(G)$. If $\Psi$ satisfies that \# $\cup_{b \in E}\{\Psi(b)\} \geqq \# E$ for every subset $E$ of $B$, then there is a permutation $\sigma:\{1, \cdots, n\} \rightarrow\{1, \cdots, n\}$ such that $g_{o(i)} \in \Psi\left(b_{i}\right)(1 \leqq i \leqq n)$.

LEMMA 3. Let $n \geqq 1$ and $0<\delta<1 / n$ be given. For $\mu \in M_{n}(X)$, take $\boldsymbol{x} \in X_{n}$ with $\varphi_{n}(\boldsymbol{x})=\mu$. Then for every $\nu \in M_{n}(X)$ with $\widetilde{d}(\mu, \nu)<\delta$ there is $\boldsymbol{y} \in X_{n}$ such that $\varphi_{n}(\boldsymbol{y})=\nu$ and $d_{n}(\boldsymbol{x}, \boldsymbol{y})<\delta$.

ProOF. Take $\delta_{0}>0$ with $\tilde{d}(\mu, \nu)<\delta_{0}<\delta \quad(<1 / n)$. We express $\boldsymbol{x}=$ $\left(x_{1}, \cdots, x_{n}\right) \in X_{n}$ and $\nu=(1 / n) \sum_{i=1}^{n} \pi\left(z_{i}\right)$. To distinguish between $x_{i}$ or $z_{i}$ $(1 \leqq i \leqq n)$ we put $S=\{1, \cdots, n\}$ and $x_{i}^{\prime}=\left(x_{i}, i\right), z_{i}^{\prime}=\left(z_{i}, i\right) \in X \times S(1 \leqq i \leqq n)$. For $B=\left\{x_{i}^{\prime}, \cdots, x_{n}^{\prime}\right\}$ and $G=\left\{z_{i}^{\prime}, \cdots, z_{n}^{\prime}\right\}$ we define a map $\Psi: B \rightarrow P(G)$ by $\Psi\left(x_{i}^{\prime}\right)=\left\{z_{j}^{\prime} \in G: d\left(\tau\left(x_{i}^{\prime}\right), \tau\left(z_{j}^{\prime}\right)\right) \leqq \delta_{0}\right\} \quad(1 \leqq i \leqq n)$ where $P(G)$ is the family of subsets of $G$ and $\tau: X \times S \rightarrow X$ is a natural projection. In order to apply 
Lemma 2 we show that \# $\cup_{x_{i}^{\prime} \in E} \Psi\left(x_{i}^{\prime}\right) \geqq E$ for every subset $E$ of $B$. Indeed, by $\widetilde{d}(\mu, \nu)<\delta_{0}$, it follows that $\nu\left((\tau E)^{\delta_{0}}\right) \geqq \mu(\tau E)-\delta_{0}$ where $(\tau E)^{\delta_{0}}=\{x \in X$ : $\left.d(x, \tau E) \leqq \delta_{0}\right\}$. Since $\mu(\tau E)=\# E / n$ and $\delta_{0}<1 / n$, we have $\nu\left((\tau E)^{\delta_{0}}\right)>(1 / n)$ (\#E-1). For every Borel set $A \subset X, \nu(A)=(1 / n)\left(\sum_{t=1}^{n} \pi\left(z_{t}\right)\right)(A)=k / n$ for some $0 \leqq k \leqq n$, so $(1 / n)\left(\sum_{i=1}^{n} \pi\left(z_{i}\right)(\tau E)^{\delta_{0}}\right) \geqq \# E / n$. Since $\# \cup_{x_{i}^{\prime} \in E}\left\{\Psi\left(x_{i}^{\prime}\right)\right\}=$ $\#\left\{z_{j}^{\prime} \in G: d\left(\tau x_{i}^{\prime}, \tau z_{j}^{\prime}\right) \leqq \delta_{0}, x_{i}^{\prime} \in E\right\}=\sum_{i=1}^{n} \pi\left(z_{i}\right)\left((\tau E)^{\delta_{0}}\right)$, we get $\# \cup_{x_{i}^{\prime} \in E}\left\{\Psi\left(x_{i}^{\prime}\right)\right\} \geqq \# E$.

Hence by Lemma 2 there is a permutation $\sigma: S \rightarrow S$ with $\Psi\left(x_{i}^{\prime}\right)=z_{\sigma(i)}^{\prime}$ $(1 \leqq i \leqq n)$. Put $y_{i}=z_{o(i)}$ and $y=\left(y_{1}, \cdots, y_{n}\right) \in X_{n}$. Then we have $\varphi_{n}(y)=$ $(1 / n) \sum_{i=1}^{n} \pi\left(y_{i}\right)=(1 / n) \sum_{i=1}^{n} \pi\left(z_{\sigma(i)}\right)=\nu$ and $d_{n}(x, y)=\max _{1 \leq i \leq n} d\left(x_{i}, z_{\sigma(i)}\right)=$ $\max _{1 \leq i \leq n} d\left(\tau x_{i}^{\prime}, \tau z_{\sigma(i)}^{\prime}\right) \leqq \delta_{0}<\delta$.

LEMMA 4. (1) If $(X, T)$ has the P.O.T.P., then $\left(X_{n}, T_{n}\right)$ has the P.O.T.P. for every $n \geqq 1$. More precisely, given $\varepsilon>0$ if there is $\delta>0$ such that every $\delta$-pseudo orbit of $T$ is $\varepsilon$-traced, then every $\delta$-pseudo orbit of $T_{n}$ is e-traced.

(2) If $(X, T)$ is tracing, then so is $\left(X_{n}, T_{n}\right)$.

Proof. ( 1 ): Let $\varepsilon>0$ be given and $\delta>0$ be a number decided by the P.O.T.P. of $(X, T)$ corresponding with $\varepsilon$. Let $\left\{\dot{x}^{k}\right\}_{k=-\infty}^{\infty}$ be a $\delta$-pseudo orbit of $\left(X_{n}, T_{n}\right)$. If one denotes $x^{k}=\left(x_{1}^{k}, \cdots, x_{n}^{k}\right)(k \in Z)$ then $\left\{x_{i}^{k}\right\}_{k=-\infty}^{\infty}$ $(1 \leqq i \leqq n)$ is a $\delta$-pseudo orbit of $(X, T)$ because $d\left(T x_{i}^{k}, x_{i}^{k+1}\right) \leqq d_{n}\left(T_{n} x^{k}, x^{k+1}\right)<\delta$ $(1 \leqq i \leqq n, k \in Z)$. By assumption, there is $y_{i} \in X(1 \leqq i \leqq n)$ with $d\left(T^{k} y_{i}, x_{i}^{k}\right) \leqq \varepsilon$ $(k \in Z)$. Put $\boldsymbol{y}=\left(y_{1}, \cdots, y_{n}\right)$ then $d_{n}\left(T_{n}^{k} y, x^{k}\right)=\max _{1 \leq i \leq n} d\left(T^{k} y_{i}, x_{i}^{k}\right) \leqq \varepsilon ;$ i.e. $\boldsymbol{y} \in X_{n} \varepsilon$-traces $\left\{\boldsymbol{x}^{k}\right\}_{k=-\infty}^{\infty}$.

(2): Let a sequence $\left\{x^{k}\right\}_{k=0}^{\infty}$ of $X_{n}$ satisfy that $\lim _{k \rightarrow \infty} d_{n}\left(T_{n} x^{k}, x^{k+1}\right)=0$. If one denotes $x^{k}=\left(x_{1}^{k}, \cdots, x_{n}^{k}\right)(k \in Z)$ then it follows that $\lim _{k \rightarrow \infty} d\left(T x_{i}^{k}\right.$, $\left.x_{i}^{k+1}\right) \leqq \lim _{k \rightarrow \infty} d_{n}\left(T_{n} x^{k}, x^{k+1}\right)=0 \quad(1 \leqq i \leqq n), \quad$ so there is $y_{i} \in X \quad$ with $\lim _{k \rightarrow \infty} d\left(T^{k} y_{i}, x_{i}^{k}\right)=0$. Putting $y=\left(y_{1}, \cdots, y_{n}\right)$ we have $d_{n}\left(T_{n}^{k} y, x^{k}\right)=$ $\max _{1 \leq i \leq n} d\left(T^{k} y_{i}, x_{i}^{k}\right) \rightarrow 0(k \rightarrow \infty)$. This proves the lemma.

Proof of Theorem 1. ( 1 ): Let $n \geqq 1$ and $\varepsilon>0$ be given. By assumption there is $0<\delta<1 / n$ such that every $\delta$-pseudo orbit of $(X, T)$ is $\varepsilon / 2$-traced. Then, by Lemma $4(1)$, every $\delta$-pseueo ordit of $\left(X_{n}, T_{n}\right)$ is $\varepsilon / 2$-traced. At first we show that for every $m>0$, every finite $\delta$-pseudo orbit $\left\{\nu_{k}\right\}_{k=0}^{m}$ of $\left(M_{n}(X), \widetilde{T}\right)$ is $\varepsilon / 2$-traced. Since $\varphi_{n}$ is surjective there is $\boldsymbol{x}^{0}=\left(x_{1}^{0}, \cdots, x_{n}^{0}\right) \in X_{n}$ with $\varphi_{n}\left(x^{0}\right)=\nu_{0}$. Then, since $\widetilde{d}\left(\widetilde{T} \nu_{0}, \nu_{1}\right)<\delta$, by Lemma 3 we can find $x^{1}=\left(x_{1}^{1}, \cdots, x_{n}^{1}\right) \in X_{n}$ such that $\varphi_{n}\left(x^{1}\right)=\nu_{1}$ and $d_{n}\left(T_{n} x^{0}, x^{1}\right)<\delta$. As $\widetilde{d}\left(\widetilde{T} \nu_{1}, \nu_{2}\right)<\delta$, by Lemma 3 we have again $x^{2}=\left(x_{1}^{2}, \cdots, x_{n}^{2}\right) \in X_{n}$ such that $\varphi_{n}\left(\boldsymbol{x}^{2}\right)=\nu_{2}$ and $d_{n}\left(T_{n} x^{1}, x^{2}\right)<\delta$. Repeated this process we get a $\delta$-pseudo orbit $\left\{x^{k}\right\}_{k=0}^{m}$ of $\left(X_{n}, T_{n}\right)$ with $\varphi_{n}\left(x^{k}\right)=\nu_{k}(0 \leqq k \leqq m)$. Hence there is $\boldsymbol{y}=$ $\left(y_{1}, \cdots, y_{n}\right) \in X_{n}$ which $\varepsilon / 2$-traces $\left\{x^{k}\right\}_{k=0}^{m}$. Put $\mu=(1 / n) \sum_{i=1}^{n} \pi\left(y_{i}\right) \in M_{n}(X)$, then we have 


$$
\widetilde{d}\left(\widetilde{T}^{k} \mu, \nu_{k}\right)=\widetilde{d}\left(\varphi_{n}\left(T_{n}^{k} \boldsymbol{y}\right), \varphi_{n}\left(\boldsymbol{x}^{k}\right)\right) \leqq d_{n}\left(T_{n}^{k} \boldsymbol{y}, \boldsymbol{x}^{k}\right) \leqq \varepsilon / 2 \quad(\text { by Lemma 1) ; }
$$

i.e. $\left\{\nu_{k}\right\}_{k=0}^{m}$ is $\varepsilon / 2$-traced by $\mu$.

Now let $\left\{\nu_{k}\right\}_{k=-\infty}^{\infty}$ be a $\delta$-pseudo orbit of $\left(M_{n}(X), \widetilde{T}\right)$. For every $m>0$ let us put $\nu_{j}^{\prime}=\nu_{j-m}(0 \leqq j \leqq 2 m)$. Then by the above argument there is $\mu_{m}^{\prime} \in M_{n}(X)$ which $\varepsilon / 2$-traces $\left\{\nu_{j}^{\prime}\right\}_{j=0}^{2 m}$. Put $\mu_{m}=\widetilde{T}^{m} \mu_{m}^{\prime}$ and take a limit point $\mu$ of sequence $\mu_{m}$. Then, since for every $k \in Z$ there is $m>|k|$ so that $\widetilde{d}\left(\widetilde{T}^{k} \mu_{m}, \widetilde{T}^{k} \mu\right) \leqq \varepsilon / 2$, we get $\widetilde{d}\left(\widetilde{T}^{k} \mu, \nu_{k}\right) \leqq \widetilde{d}\left(\widetilde{T}^{k} \mu, \widetilde{T}^{k} \mu_{m}\right)+\widetilde{d}\left(\widetilde{T}^{k+m} \mu_{m}^{\prime}, \nu_{k+m}^{\prime}\right) \leqq \varepsilon / 2+$ $\varepsilon / 2=\varepsilon$. This shows that $\left(M_{n}(X), \widetilde{T}\right)$ has the P.O.T.P..

(2): Let $n \geqq 1$ be given and $\left\{\nu_{k}\right\}_{k=0}^{\infty}$ be a sequence of $M_{n}(X)$ with $\lim _{k \rightarrow \infty} \widetilde{d}\left(\widetilde{T} \nu_{k}, \nu_{k+1}\right)=0$. Without loss of generality we may assume $\widetilde{d}\left(\widetilde{T} \nu_{k}, \nu_{k+1}\right)<1 / n(k \geqq 0)$. By same argument as in the proof of (1) we get a sequence $\left\{x^{k}\right\}_{k=0}^{\infty}$ of $X_{n}$ such that $\varphi_{n}\left(x^{k}\right)=\nu_{k}(k \geqq 0)$ and $\lim _{k \rightarrow \infty} d_{n}\left(T_{n} x^{k}\right.$, $\left.\boldsymbol{x}^{k+1}\right)=0$. Since $\left(X_{n}, T_{n}\right)$ is tracing (by Lemma $4(2)$ ), there is $x \in X_{n}$ with $\lim _{k \rightarrow \infty} d_{n}\left(T_{n}^{k} x, x^{k}\right)=0$. Then for $\mu=\varphi_{n}(x)$ it follows that $\tilde{d}\left(\widetilde{T}^{k} \mu, \nu_{k}\right)=$ $\widetilde{d}\left(\varphi_{n}\left(T_{n}^{k} x\right), \varphi_{n}\left(x^{k}\right)\right) \leqq d_{n}\left(T_{n}^{k} x, x^{k}\right) \rightarrow 0(k \rightarrow \infty)$. The proof is completed.

\section{§3. Proof of Theorem 2.}

We shall prove Theorem 2 by a series of lemmas.

LEMMA 5. Let $\mu, \nu$ and $\mu_{i}(i=1,2)$ belong to $M(X)$.

(1) $\widetilde{d}(\alpha \mu+(1-\alpha) \nu, \beta \mu+(1-\beta) \nu) \leqq \beta-\alpha$ for $0 \leqq \alpha \leqq \beta \leqq 1$.

(2) $\tilde{d}\left(\alpha \mu_{1}+(1-\alpha) \mu_{2}, \nu\right) \leqq \max \left\{\widetilde{d}\left(\mu_{1}, \nu\right), \widetilde{d}\left(\mu_{2}, \nu\right)\right\}$ for $0 \leqq \alpha \leqq 1$.

Proof. (1): Let a Borel set $A \subset X$ be given. Then we calculate

$$
\begin{aligned}
(\beta \mu+ & (1-\beta) \nu)\left(A^{\beta-\alpha}\right)+(\beta-\alpha)-(\alpha \mu+(1-\alpha) \nu)(A) \\
= & \alpha\left(\mu\left(A^{\beta-\alpha}\right)-\mu(A)\right)+(1-\alpha)\left(\nu\left(A^{\beta-\alpha}\right)-\nu(A)\right) \\
& +(\beta-\alpha)\left(1+\mu\left(A^{\beta-\alpha}\right)-\nu\left(A^{\beta-\alpha}\right)\right) \geqq 0
\end{aligned}
$$

where $A^{\beta-\alpha}=\cup_{x \in A}\{y \in X: d(x, y) \leqq \beta-\alpha\}$. From this we have conclusion.

(2): Put $c=\max \left\{\widetilde{d}\left(\mu_{1}, \nu\right), \widetilde{d}\left(\mu_{2}, \nu\right)\right\}$ and take $\varepsilon>c$. For every Borel set $A \subset X$, since $\mu_{i}\left(A^{*}\right)+\varepsilon-\nu(A) \geqq 0(i=1,2)$, we have

$$
\begin{aligned}
& \left(\alpha \mu_{1}+(1-\alpha) \mu_{2}\right)\left(A^{e}\right)+\varepsilon-\nu(A) \\
& \quad=\alpha\left(\mu_{1}\left(A^{\varepsilon}\right)+\varepsilon-\nu(A)\right)+(1-\alpha)\left(\mu_{2}\left(A^{\varepsilon}\right)+\varepsilon-\nu(A)\right) \geqq 0 .
\end{aligned}
$$

Therefore $\widetilde{d}\left(\alpha \mu_{1}+(1-\alpha) \mu_{2}, \nu\right) \leqq \inf \{\varepsilon: c<\varepsilon\}=c$, proving the lemma.

LEMMA 6. Let $T$ be a homeomorphism of $X$ and $\widetilde{T}$ an induced homeomorphism of $M(X)$. Then $(M(X), \widetilde{T})$ is $\widetilde{T}$-connected.

Proof. Let $\mu, \nu \in M(X)$ and $\alpha>0$ be given. Take $n \geqq 1$ with $1 / n<\alpha$. 
For $0 \leqq i \leqq n$ let us define $\mu_{i}$ and $\nu_{i}$ by $\mu_{i}=(1-(i / n)) \widetilde{T}^{i} \mu+(i / n) \widetilde{T}^{i-n} \nu$ and $\nu_{i}=(1-(i / n)) \widetilde{T}^{i} \nu+(i / n) \widetilde{T}^{i-n} \mu$. Obviously $\mu_{0}=\mu=\nu_{n}$ and $\nu_{0}=\nu=\mu_{n}$. By Lemma 5(1) we have

$$
\begin{aligned}
\widetilde{d}\left(\widetilde{T} \mu_{i}, \mu_{i+1}\right) & =\widetilde{d}\left(\left(1-\frac{i}{n}\right) \widetilde{T}^{i+1} \mu+\frac{i}{n} \widetilde{T}^{i+1-n} \nu,\left(1-\frac{i+1}{n}\right) \widetilde{T}^{i+1} \mu+\frac{i+1}{n} \widetilde{T}^{i+1-n} \nu\right) \\
& \leqq \frac{i+1}{n}-\frac{i}{n}<\alpha \quad(0 \leqq i \leqq n-1)
\end{aligned}
$$

and similarly $\tilde{d}\left(\widetilde{T}_{i}, \nu_{i+1}\right)<\alpha(0 \leqq i \leqq n-1)$. This proves the lemma.

LEMma 7. If $(X, T)$ is tracing and T-connected, then $(X, T)$ has the P.O.T.P..

Proof. Suppose the lemma is false. Then there is $\varepsilon>0$ such that for every $k \geqq 1$ there is a $(1 / k)$-pseudo orbit $\left\{x_{1}^{k}, \cdots, x_{N_{k}}^{k}\right\}$ of $(X, T)$ such that there is no $z \in X$ with $d\left(T^{j} z, x_{j}^{k}\right) \leqq \varepsilon\left(1 \leqq j \leqq N_{k}\right)$. By $T$-connectedness, there is a (1/k)-pseudo orbit $\left\{z_{0}^{k}, \cdots, z_{L_{k}}^{k}\right\}$ with $z_{0}^{k}=x_{N_{k}}^{k}$ and $z_{L_{k}}^{k}=x_{1}^{k+1}(k \geqq 1)$. Renewing the indices of a sequence

$$
\left\{\cdots, x_{1}^{k}, \cdots, x_{N_{k}}^{k}, z_{1}^{k}, \cdots, z_{N_{k-1}}^{k}, x_{1}^{k+1}, \cdots\right\},
$$

we have a sequence $\left\{x_{n}\right\}_{n=0}^{\infty}$ which satisfies $\lim _{n \rightarrow \infty} d\left(T x_{n}, x_{n+1}\right)=0$. Since $(X, T)$ is tracing, there is $z \in X$ with $\lim _{n \rightarrow \infty} d\left(T^{n} z, x_{n}\right)=0$. Therefore for some $k>0,\left\{x_{i}^{k}\right\}_{i=1}^{N_{k}}$ is $\varepsilon$-traced. This is a contradiction.

If $(M(X), \widetilde{T})$ is tracing, by Lemmas 6 and $7,(M(X), \widetilde{T})$ has the P.O.T.P.. This prove Theorem 2(1). Next we show Theorem 2(2).

Lemma 8. Assume $(X, T)$ is T-connected and has the P.O.T.P.. If the set of fixed points under $T$ is nonempty, then $(X, T)$ is topologically mixing.

Proof. Let $U, V \subset X$ be nonempty open sets. There are $x \in U, y \in V$ and $\varepsilon>0$ such that $B(x ; \varepsilon) \subset U$ and $B(y ; \varepsilon) \subset V$, where $B(z ; \varepsilon)=\left\{z^{\prime} \in X\right.$ : $\left.d\left(z, z^{\prime}\right) \leqq \varepsilon\right\}$. Since $(X, T)$ has the P.O.T.P., there is $\delta>0$ so that every $\delta$-pseudo orbit of $(X, T)$ is $\varepsilon$-traced. Take a fixed point $p \in X$ under $T$. By $T$-connectedness, there are $\delta$-pseudo orbits $\left\{x_{i}\right\}_{i=0}^{a}$ and $\left\{y_{i}\right\}_{i=0}^{b}$ such that $x_{0}=x$, $x_{a}=p=y_{0}$ and $y_{b}=y$. Put $N=a+b+1 \geqq 0$. Given $n \geqq N$, since a sequence

$$
\left\{x_{0}, x_{1}, \cdots, x_{a}, p, \cdots, p\left(n-N \text { times), } y_{0}, \cdots, y_{b}\right\}\right.
$$

is a $\delta$-pseudo orbit, there is $z \in X$ which $\varepsilon$-traces this sequence. As $d(x, z)=d\left(x_{0}, z\right) \leqq \varepsilon$ and $d\left(y, T^{n} z\right)=d\left(y_{b}, T^{n} z\right) \leqq \varepsilon$, we have $z \in B(x ; \varepsilon) \cap$ $T^{-n} B(y ; \varepsilon) \subset U \cap T^{-n} V$; i.e. $(X, T)$ is topologically mixing. 
LEMMA 9. If $(X, T)$ is topologically mixing and has the P.O.T.P., then $(X, T)$ satisfies weak specification.

Proof. Let $\varepsilon>0$ be given. There is $\delta>0$ such that every $\delta$-pseudo orbit is $\varepsilon$-traced. By topological mixing and the compactness of $X$, there is $M>0$ such that for every $x, y \in X, T^{n} B(x ; \delta) \cap B(y ; \delta) \neq \varnothing$ for every $n \geqq M$, where $B(z ; \delta)=\left\{z^{\prime} \in X: d\left(z, z^{\prime}\right) \leqq \delta\right\}$. For every $k \geqq 1$, let $k$ points $x_{1}, \cdots, x_{k} \in X$ and a set of integers $a_{1} \leqq b_{1}<\cdots<a_{k} \leqq b_{k}$ with $a_{i+1}-$ $b_{i} \geqq M(1 \leqq i \leqq k-1)$ be given. By choice of $M$, there are $y_{i} \in B\left(T^{b_{i}} x_{i} ; \delta\right)$ $(1 \leqq i \leqq k-1)$ with $T^{a_{i+1}-b_{i}} y_{i} \in B\left(T^{a_{i+1}} x_{i+1} ; \delta\right)$. Then a sequence $\left\{T^{a_{1}} x_{1}, \cdots\right.$, $\left.T^{b_{1}-1} x_{1}, y_{1}, \cdots, T^{a_{2}-b_{1}} y_{1}, T^{a_{2}} x_{2}, \cdots, T^{b_{k}} x_{k}\right\}$ is $\delta$-pseudo orbit, so there is $z \in X$ with $d\left(T^{n} z, T^{n} x_{i}\right) \leqq \varepsilon\left(a_{i} \leqq n \leqq b_{i}, 1 \leqq i \leqq k\right)$. This proves the Lemma.

We remarked in $\S 1$ that the set of fixed points of $(M(X), \widetilde{T})$ is nonempty. Hence if $(M(X), \widetilde{T})$ has the P.O.T.P., by Lemmas 6, 8 and 9, $(M(X), \widetilde{T})$ satisfies weak specification. Therefore the proof of Theorem $2(2)$ is completed by the following lemma.

LEMMA 10. If $(M(X), \widetilde{T})$ satisfies weak specification, then $(M(X), \widetilde{T})$ satisfies specification.

Proof. Let $\varepsilon>0$ be given and $M=M(\varepsilon)>0$ an integer determined from the definition of weak specification. For every $k \geqq 1$, let $k$ points $\mu_{1}, \cdots, \mu_{k} \in M(X)$ and a set of integers $a_{1} \leqq b_{1}<\cdots<a_{k} \leqq b_{k}$ with $a_{i+1}-b_{i} \geqq M$ $(1 \leqq i \leqq k-1)$ and $p \geqq b_{k}-a_{1}+M$ be given. Using weak specification, we can find a sequence $\nu_{j} \in M(X)(j=1,2, \cdots)$ such that $\widetilde{d}\left(\widetilde{T}^{n+m p} \nu_{j}, \widetilde{T}^{n} \mu_{i}\right) \leqq \varepsilon$ $\left(a_{i} \leqq n \leqq b_{i}, 1 \leqq i \leqq k, 0 \leqq m \leqq j\right)$. Take a limit point $\nu$ of the sequence $\nu_{j}$. Then it follows that $\widetilde{d}\left(\widetilde{T}^{n+m}{ }^{2}, \widetilde{T}^{n} \mu_{j}\right) \leqq \varepsilon \quad\left(a_{i} \leqq n \leqq b_{i}, 1 \leqq i \leqq k, m \geqq 0\right)$. Put $\mu=\lim _{s \rightarrow \infty}(1 / s) \sum_{m=0}^{s-1} \widetilde{T}^{m p} \nu$ for some $\{s\}$. By Lemma $5(2)$, we have for $a_{i} \leqq n \leqq b_{i}(1 \leqq i \leqq k)$,

$$
\begin{aligned}
& \widetilde{d}\left(\widetilde{T}^{n} \mu, \widetilde{T}^{n} \mu_{i}\right)=\lim _{s \rightarrow \infty} \widetilde{d}\left((1 / s) \sum_{m=0}^{s-1} \widetilde{T}^{n+m p} \nu, \widetilde{T}^{n} \mu_{i}\right) \\
& \quad \leqq \lim _{s \rightarrow \infty} \max \left\{\widetilde{d}\left(\widetilde{T}^{n+m p} \nu, \widetilde{T}^{n} \mu_{i}\right) ; 0 \leqq m \leqq s-1\right\} \leqq \varepsilon .
\end{aligned}
$$

Also, by Lemma 5(1), we have $\tilde{d}\left(\widetilde{T}^{p} \mu, \mu\right) \leqq \lim _{s \rightarrow \infty}\left\{\widetilde{d}\left(s^{-1} \sum_{m=1}^{s} \widetilde{T}^{m p} \nu,(s-1)^{-1} \times\right.\right.$ $\left.\left.\sum_{m=1}^{s-1} \widetilde{T}^{m p} \nu\right)+\widetilde{d}\left((s-1)^{-1} \sum_{m=1}^{s-1} \widetilde{T}^{m p} \nu, s^{-1} \sum_{m=0}^{s-1} \widetilde{T}^{m p} \nu\right)\right\} \leqq \lim _{s \rightarrow \infty} 2 / s=0$, hence $\widetilde{T}^{p} \mu=\mu$. This completes the proof.

\section{References}

[1] W. BAUER and K. Sigmund, Topological dynamics of transformations induced on the space of probability measures, Monatsh. Math., 79 (1975), 81-92. 
[2] R. BowkN, $\omega$-limit sets for Axiom A diffeomorphisms, J. Differential Equations, 18 (1975), 333-339.

[3] M. Denker, C. Grillennberger and K. Sigmund, Ergodic Theory on Compact Spaces, Lecture Notes Math., 527, Springer, Berlin-Heidelberg-New York, 1964.

[4] P. Halmos and H. Vaughn, The marriage problem, Amer. J. Math., 72 (1950), 214-215.

[5] K. R. Parthasarathy, Probability Measures on Metric Spaces, Academic Press, New York and London, 1967.

[6] K. Sigmund, On minimal centers of attraction and generic points, J. Reine Angew. Math., 295 (1977), 72-79.

[7] V. Sterassen, The existence of probability measures with given marginals, Ann. Math. Statistics, 36 (1965), 423-439.

\author{
Present Address: \\ DePARTMENT OF MATHEMATICS \\ FACULTY OF ScIENCES \\ TOKYO METROPOLITAN UNIVERSITY \\ Fukazawa, Setagaya-ro, Tokyo 158
}

